\title{
Electroless Deposited Gold Nanoparticles on Glass Plates as Sensors for Measuring the Dielectric Constant of Solutions
}

\author{
Y. Kobayashi and Y. Ishii \\ Department of Biomolecular Functional Engineering, College of Engineering, Ibaraki University, 4-12-1 Naka-narusawa-cho, Ibaraki, \\ Hitachi 316-8511, Japan \\ Correspondence should be addressed to Y. Kobayashi; ykoba@mx.ibaraki.ac.jp
}

Received 29 November 2012; Accepted 7 March 2013

Academic Editor: Frank Hubenthal

Copyright ( 2013 Y. Kobayashi and Y. Ishii. This is an open access article distributed under the Creative Commons Attribution License, which permits unrestricted use, distribution, and reproduction in any medium, provided the original work is properly cited.

\begin{abstract}
This work describes a method for the deposition of Au nanoparticles on glass plates (Au-glass). An electroless metal plating technique was extended to the Au nanoparticle deposition. The technique consisted of three steps that took place on the glass plate: (1) adsorption of $\mathrm{Sn}^{2+}$ ions, (2) deposition of metallic $\mathrm{Ag}$ nuclei generated by reducing $\mathrm{Ag}^{+}$ions with $\mathrm{Sn}^{2+}$ ions on the $\mathrm{Sn}$ adsorbed sites, and (3) deposition of $\mathrm{Au}$ nanoparticles by reducing $\mathrm{Au}^{+}$ions on the $\mathrm{Ag}$ surface. TEM observation revealed that metallic Au nanoparticles with a size of $34.6 \pm 11.5 \mathrm{~nm}$ were formed on the glass surface. A surface plasmon resonance absorption peak was observed, and its peak wavelength redshifted by immersing the Au-glass into a solution with a large dielectric constant. The redshift corresponded qualitatively to the calculation by the Mie theory accompanying the Drude expression, which was based on the change of the dielectric constant of the solution. The obtained results indicated that the Au-glass functioned as a sensor for measuring the dielectric constant of the solution.
\end{abstract}

\section{Introduction}

Nanometer-sized particles have special interest because they are expected to exhibit unique properties different from those of bulk material $[1,2]$, which is called "size effect." Among various nanoparticles, nanoparticles of metals such as $\mathrm{Au}$ and $\mathrm{Ag}$ show a special optical property like surface plasmon resonance (SPR) absorption, which depends on particle size and particle shape $[1,3,4]$. Since the SPR absorption is also dependent on environment around the particles such as dielectric constant $[1,3,4]$, this dependence can be used to measure dielectric constants of materials $[3,4]$.

Nanoparticles tend to aggregate, which deteriorates their unique properties derived from the size effect. Immobilizing the nanoparticles on supports such as powders and plates is a candidate to prevent the aggregation. Recently, there have been some reports on deposition of metal nanoparticles on submicron-sized particles [5-8]. Frequently, the deposition techniques involve simple mixing of a metal nanoparticle colloid solution and a suspension of submicron-sized spheres $[6,9,10]$. In this technique, however, aggregation of the nanoparticles during the deposition cannot be negligible, and it is necessary to remove excess undeposited nanoparticles out of the suspension.

An electroless metal plating technique can make metallic films plated on insulating support materials [11-13]. In our previous work, Au nanoparticles were successfully deposited on silica spheres by the technique [14]. The technique consists of three steps. The first is a modification of the surface of the supports with $\mathrm{Sn}^{2+}$ ions $\left(\mathrm{Sn}^{2+}\right.$-sensitization). The second is a redox reaction. $\mathrm{Ag}^{+}$ions get reduced into metallic $\mathrm{Ag}$ during oxidation of the $\mathrm{Sn}^{2+}$ ions to $\mathrm{Sn}^{4+}$ (Ag activation), which adheres metallic Ag nuclei to the silica surface. The third is a reduction of $\mathrm{Au}^{+}$ions into metallic $\mathrm{Au}$ on the $\mathrm{Ag}$ nuclei because of the galvanic displacement of the metallic Ag by $\mathrm{Au}$. In an early stage of the plating, generation and growth of metallic Au nuclei should take place on the activated surface, and consequently metallic Au nanoparticles are deposited on the support. This technique takes an advantage of the simple mixing method, because the reduction of $\mathrm{Au}^{+}$takes place only on the Ag sites so that the aggregation of nanoparticles during the deposition can be avoided. 


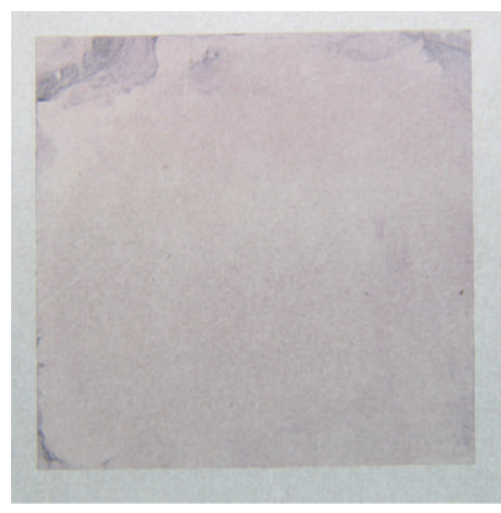

(a)

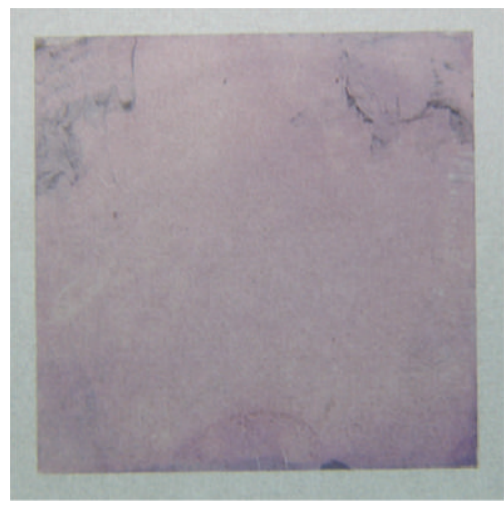

(c)

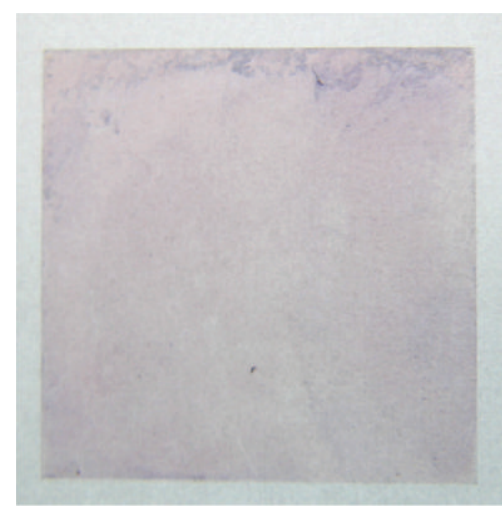

(b)

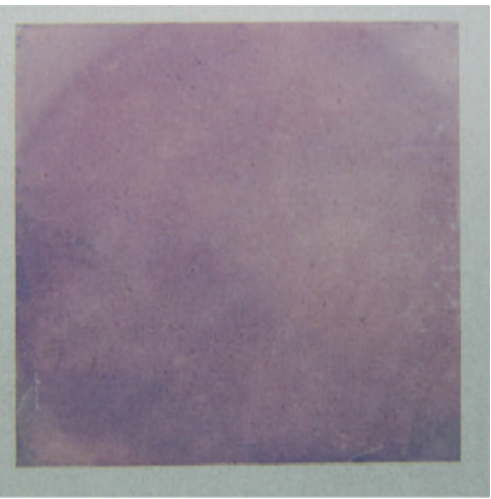

(d)

Figure 1: Photographs of Au-glass plates fabricated by electroless plating technique. The deposition times were (a) 5, (b) 10, (c) 30, and (d) 60 minutes.

The present work proposes a method for depositing Au nanoparticles on glass plates by the electroless metal plating. Their optical properties in solutions with various dielectric constants were also studied toward a simple and easy sensor measuring dielectric constants of solutions.

\section{Experimental Details}

2.1. Materials. Tin chloride (anhydrous) (97\%) and trifluoroacetic acid (98\%) were used for preparation of "Sn solution." Silver nitrate (99.8\%) and aqueous ammonia (28.0-30\%) were used for preparation of "Ag solution." Oromerse Part $\mathrm{B}$ (Au electroless plating solution), formaldehyde solution (36.0-38\%), sodium bicarbonate (99.5-100.3\%), sodium sulfite $(97 \%)$, and sulfuric acid (95\%) were used for preparation of "Au solution." Ethylene glycol (EG, 99.5\%) was used for preparing solutions with various dielectric constants. To improve affinity between ions of metals such as Sn, Ag, and $\mathrm{Au}$ and the glass plates, polyvinylpyrrolidone (PVP, $K=$ 30) was used as a pretreatment agent of the substrate. The Oromerse Part B and the other chemicals were supplied by Technic Inc. and Kanto Chemical Co., Inc., respectively, with no financial support from the two companies. All chemicals were used as received. Sodium glass plates (Matsunami Glass Ind., Ltd., $18 \times 18 \mathrm{~mm}, 0.12-0.17 \mathrm{~mm}$ thick) were used as a substrate. Water that was ion exchanged and distilled with Shimadzu SWAC-500 was used in all the preparations.

\subsection{Methods}

2.2.1. Preparation. Prior to Au deposition by the electroless metal plating, the glass plates were pretreated as follows. The glass plates were immersed in acetone for $5 \mathrm{~min}$, water for $5 \mathrm{~min}$, and then in $1 \mathrm{~g} / \mathrm{L}$ PVP aqueous solution for $24 \mathrm{~h}$. After the pretreatment, the glass plates were washed by immersing in water for $5 \mathrm{~min}$.

The electroless metal plating technique that included three steps was employed for Au deposition on the pretreated glass plates, according to a work performed by Menon and Martin [15]. The first step was performed for surface sensitization of the glass plates. Sn solution was first prepared by dissolving the tin chloride $(0.05 \mathrm{~g})$ in the water $(10 \mathrm{~mL})$ with addition of the trifluoroacetic acid $(0.05 \mathrm{~mL})$. The glass plate was immersed into the $\mathrm{Sn}$ solution, which resulted in the adsorption of $\mathrm{Sn}^{2+}$ on the plate. After $45 \mathrm{~min}$, the glass plate was washed with immersion in water $\left(\mathrm{Sn}^{2+}\right.$-glass). The second step was performed for surface activation. Ag solution was first prepared by dissolving the silver nitrate $(0.06 \mathrm{~g})$ in the water $(10 \mathrm{~mL})$ with addition of several drops of the aqueous ammonia. The addition of the first drop formed brownish fine precipitate of silver (I) oxide, and then the 
succeeding addition made the solution transparent due to formation of $\mathrm{Ag}\left(\mathrm{NH}_{3}\right)_{2}{ }^{+}$complex. The $\mathrm{Sn}^{2+}$-glass plate was immersed into the $\mathrm{Ag}$ solution, which resulted in deposition of Ag nuclei on the plate. After $5 \mathrm{~min}$, the Ag-glass was also washed with immersion in water (Ag-glass). The third step was Au plating. Au plating solution was first prepared by mixing the water $(10 \mathrm{~mL})$, the Oromerse Part B $(0.25 \mathrm{~mL})$, the formaldehyde solution $(0.5 \mathrm{~mL})$, the sodium bicarbonate $(0.021 \mathrm{~g})$, the sodium sulfite $(0.16 \mathrm{~g})$, and several drops of the sulfuric acid. The formaldehyde solution, the sodium bicarbonate, and the sodium sulfite were used for reduction of $\mathrm{Au}$ ions, $\mathrm{pH}$ buffering, and stabilization of Au complex ions, respectively. The sulfuric acid was used for adjustment of $\mathrm{pH}$ to 10.5 to control Au electroless plating rate. The Agglass plate was immersed into the Au plating solution, which resulted in deposition of Au nanoparticles on the plate. The deposition times were for 5-60 min. The obtained glass plate was also washed with immersion in water and then annealed at $300^{\circ} \mathrm{C}$ in air (Au-glass).

2.2.2. Characterization. The Au-glass plates were characterized by visible extinction spectroscopy and transmittance electron microscopy (TEM). Visible extinction spectra of the $\mathrm{Au}$-glass plates in air and mixture of EG and water were measured with a Shimadzu UV-3101PC spectrophotometer. TEM was performed with a JEOL JEM-2100 microscope operating at $200 \mathrm{kV}$. Samples for TEM were prepared by powdering the Au-glass plates with a mortar, suspending the obtained powder in water, dropping, and evaporating the suspension onto a collodion-coated copper grid.

\section{Results and Discussion}

3.1. Au Deposition. Figure 1 shows photographs of Au-glass plates by electroless metal plating technique at various deposition times. The glass plates had a purple tone, which appeared to be derived from SPR absorption of Au nanoparticles. This observation implied that Au nanoparticles were deposited on the glass plate with the present method. The purple tone was ununiform, which meant that the $\mathrm{Au}$ nanoparticles were not uniformly deposited. The present method is still required to be improved. As the deposition time increased, the purple tone of the glass plate increased. This indicated that the amount of Au deposited on the glass plate increased with increasing the deposition time.

Figure 2 shows visible extinction spectra of the Au-glass plates measured in air. Peaks were clearly observed at 520 $540 \mathrm{~nm}$ for all the samples. These peaks were attributed to the SPR absorption [1]. Accordingly, this result supported the deposition of Au nanoparticles on the glass plates. The peak intensity increased with an increase in the deposition time, which indicated that a large amount of Au nanoparticles were deposited at the long deposition times.

Figure 3 shows a TEM image of the Au-glass plate. Darker and lighter parts were determined to be Au and glass plate, respectively, due to the electron density difference between them. Au particles with a size of $34.6 \pm 11.5 \mathrm{~nm}$ were observed

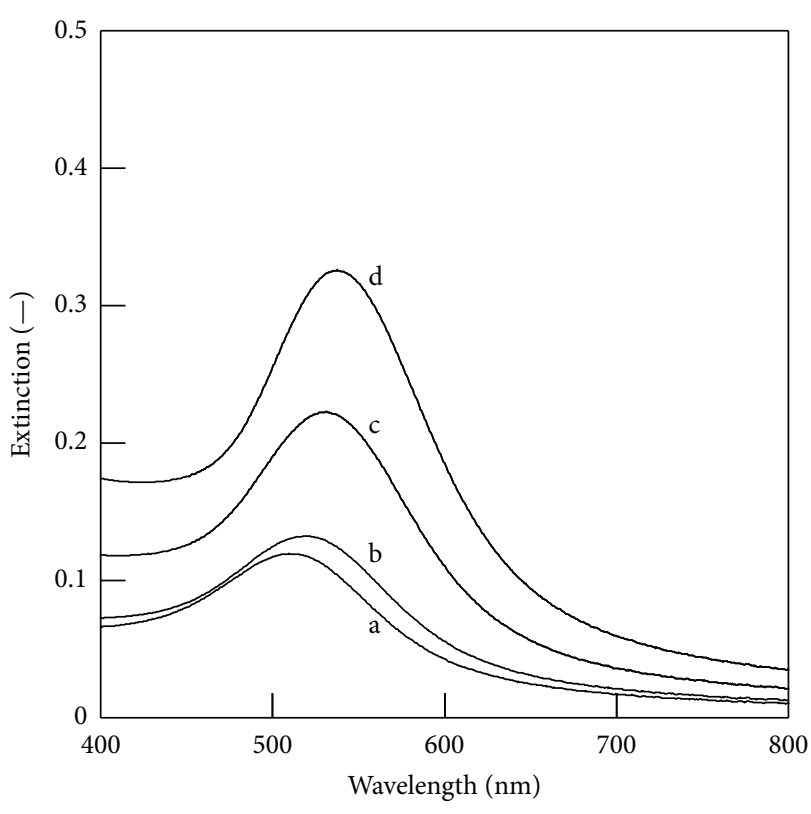

FIGURE 2: Visible extinction spectra of Au-glass plates measured in air. The samples were the same as in Figure 1.

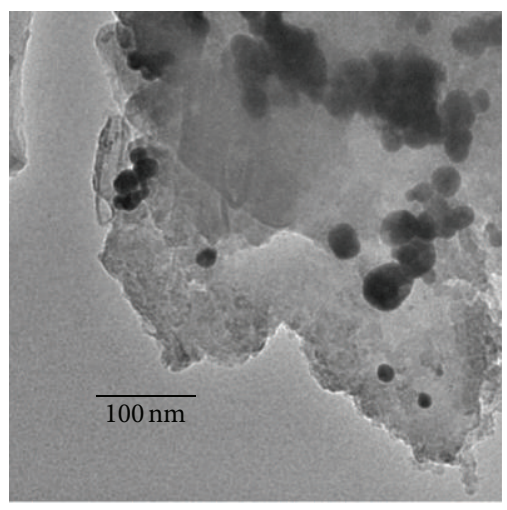

FIgure 3: TEM image of the Au-glass plate. The sample was the same as in Figure 1(d).

on the glass. This observation also supported the deposition of Au nanoparticles.

3.2. Optical Properties. Figure 4 shows visible extinction spectra of $\mathrm{Au}$-deposited glass plate in various solvents. An SPR absorption peak appeared at $534 \mathrm{~nm}$ in water. In EG/water solvents, the SPR peak position redshifted with an increase in EG content in EG/water the solvent. At an EG content of $100 \%$, that is, in EG with no water, the peak position reached $541 \mathrm{~nm}$. Dielectric constants of water and EG are 1.7769 and 2.0443, respectively. Dielectric constant of the EG/water increases in the range of 1.7769-2.0443, as the EG content in EG/water solvent increases from 0 (water) to $100 \%$ (EG). The SPR peak position was plotted versus the dielectric constant of the solvent, as shown in Figure 5. The SPR peak position increased with an increase in the dielectric constant of solvent. Several points deviated 


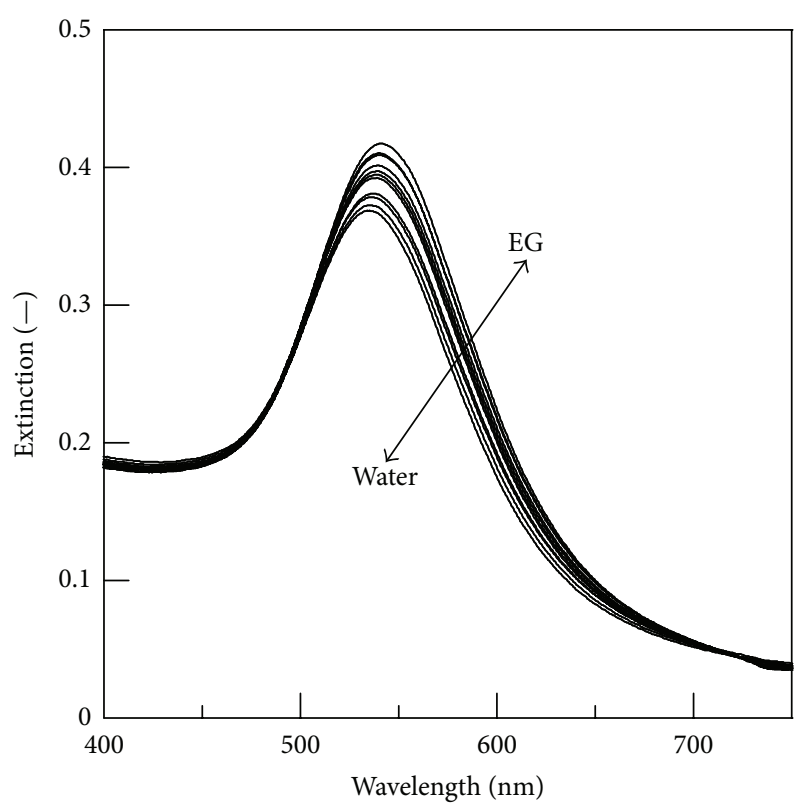

FIGURE 4: Visible extinction spectra of Au-glass plate in water, EG/water solvents with various EG contents, and EG.

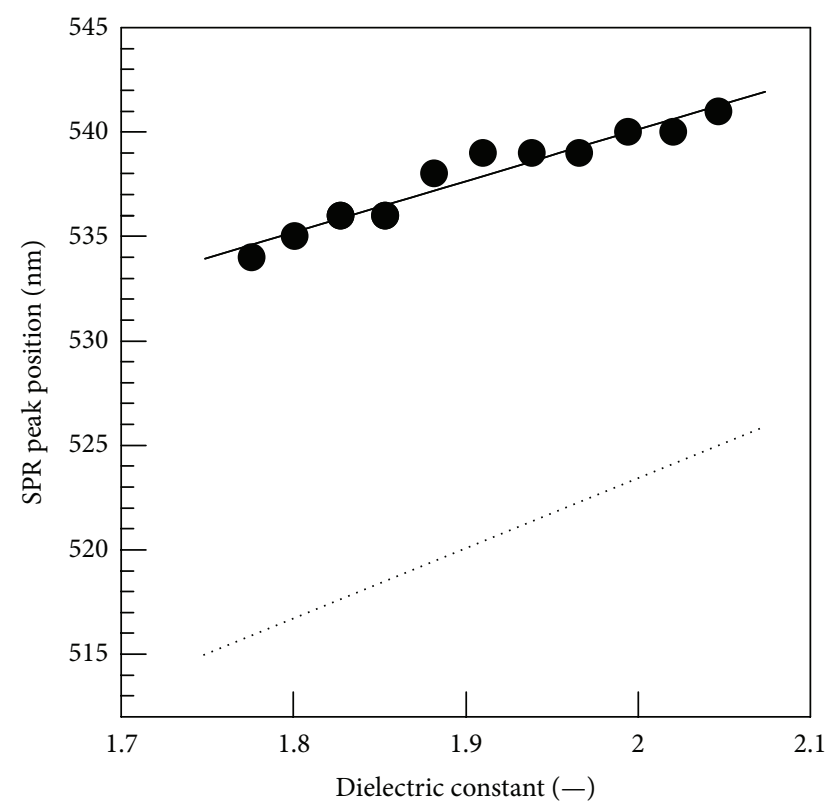

FIGURE 5: Peak position of surface plasmon resonance absorption of Au-glass plate in EG/water solvents versus dielectric constant of EG/water solvent (solid line). The Au-glass plate used was fabricated at the same conditions as the sample in Figure 1(d). The dotted line stands for the calculated line (see text).

from a correlation line that was drawn by linear fitting. The ununiform deposition of $\mathrm{Au}$ nanoparticles was considered to provide the deviation, though its precise reason is still unclear. In the case of Au nanoparticles, the redshift of SPR band is due to a local increase of refractive index or dielectric constant around the Au particles [3]. In the literature working on the Mie theory accompanying the Drude expression [16], it is assumed that those nanometer-sized particles are still accurately treated as dipole oscillators. With the assumption, the extinction cross-section, $Q_{\text {ext }}$, can be calculated from electrostatics as a sum of the absorption cross-section, $Q_{a b s}$, and the scattering cross-section, $Q_{\text {sca }}$, as follows:

$$
\begin{aligned}
Q_{\mathrm{ext}} & =Q_{\mathrm{abs}}+Q_{\mathrm{sca}} \\
& =\frac{8 \pi R \varepsilon_{m}^{1 / 2}}{\lambda} \operatorname{Im}\left[\frac{\varepsilon_{p}-\varepsilon_{m}}{\varepsilon_{p}+2 \varepsilon_{m}}\right]+\frac{128 \pi^{4} R^{4} \varepsilon_{m}^{2}}{3 \lambda^{4}}\left|\frac{\varepsilon_{p}-\varepsilon_{m}}{\varepsilon_{p}+2 \varepsilon_{m}}\right|^{2},
\end{aligned}
$$

where $R, \varepsilon$, and $\lambda$ stand for the coated particle radius, the dielectric function, and the wavelength, respectively [17]. The subscripts $p$ and $m$ refer to the particles (Au nanoparticles) and the medium material (EG/water solvent), respectively. $\varepsilon_{p}$ was calculated with the Drude expression. Using parameters taken from [16], extinction spectra were drawn, which determined SPR peak positions for dielectric constant of solvent. This calculated line is also shown in Figure 5. A tendency for the redshift of the experimental peak position with the increase in dielectric constant of solvent could be in qualitative represented with the calculations, which supported that a mechanism similar to the Mie theory accompanying the Drude expression was also considered in the present work. There was large difference in SPR peak position between the experimental data and the calculated values. In this calculation, the Au nanoparticles were assumed to be dispersed in medium. In contrary, the Au nanoparticles were immobilized on the surface of glass plate in the present work. Accordingly, the dielectric constant around the $\mathrm{Au}$ nanoparticles should have been strongly influenced by the glass. This influence probably provided the difference in the SPR peak positions. The theory used is required to be modified to accurately explain the difference. The obtained results indicated that the Au-glass plates fabricated in the present work functioned as a sensor that could measure the dielectric constant of solvent. However, a few subject matters to be solved were found by performing the present work, which have been made one of our future work.

\section{Conclusions}

$\mathrm{Au}$ nanoparticles were deposited on the surfaces of glass plates employing the electroless metal plating technique consisted of three steps. In the first step (surface sensitization), $\mathrm{Sn}^{2+}$ ions were adsorbed on the glass surface. In the second step (surface activation), $\mathrm{Ag}^{+}$ions were reduced on the surface-sensitized sites, which resulted in the deposition of metallic Ag nuclei. In the third step, $\mathrm{Au}^{+}$ions were reduced on the surface-activated sites, which resulted in the deposition of metallic Au nanoparticles. The deposited metallic Au nanoparticles had the size of ca. $35 \mathrm{~nm}$. The peak wavelength of surface plasmon resonance absorption of Au nanoparticles for the Au-glass plate immersed in EG/water solution redshifted with the increase in EG content of EG/water solution. The shift was qualitatively explained with the Mie theory accompanying the Drude expression based on the change of dielectric constant of the solution. Accordingly, the Au-glass 
plate produced in the present work was expected to be used as a sensor for measuring the dielectric constant of the solution.

\section{Conflict of Interests}

The Oromerse Part B, the other chemicals, and the sodium glass plates were supplied by Technic Inc., Kanto Chemical Co., Inc., and Matsunami Glass Ind., Ltd., respectively, with no financial support from the three companies.

\section{Acknowledgment}

The authors express their thanks to Professor T. Noguchi at the College of Science of Ibaraki University, Japan, for his support in TEM observations.

\section{References}

[1] S. Basu, S. K. Ghosh, S. Kundu et al., "Biomolecule induced nanoparticle aggregation: effect of particle size on interparticle coupling," Journal of Colloid and Interface Science, vol. 313, no. 2, pp. 724-734, 2007.

[2] L. Wang, W. Ma, L. Xu et al., "Nanoparticle-based environmental sensors," Materials Science and Engineering R, vol. 70, no. 36, pp. 265-274, 2010.

[3] T. Som and B. Karmakar, "Plasmon tuning of nano-Au in dichroic devitrified antimony glass nanocomposites by refractive index control," Chemical Physics Letters, vol. 479, no. 1-3, pp. 100-104, 2009.

[4] A. Chhatre, P. Solasa, S. Sakle, R. Thaokar, and A. Mehra, "Color and surface plasmon effects in nanoparticle systems: case of silver nanoparticles prepared by microemulsion route," Colloids and Surfaces A, vol. 404, pp. 83-92, 2012.

[5] M. A. Nadeem, M. Murdoch, G. I. N. Waterhouse et al., "Photoreaction of ethanol on $\mathrm{Au} / \mathrm{TiO}_{2}$ anatase: comparing the micro to nanoparticle size activities of the support for hydrogen production," Journal of Photochemistry and Photobiology A, vol. 216, no. 2-4, pp. 250-255, 2010.

[6] B. J. Jankiewicz, D. Jamiola, J. Choma, and M. Jaroniec, "Silicametal core-shell nanostructures," Advances in Colloid and Interface Science, vol. 170, no. 1-2, pp. 28-47, 2012.

[7] J. Xue, C. Wang, and Z. Ma, "A facile method to prepare a series of $\mathrm{SiO}_{2} @ \mathrm{Au}$ core/shell structured nanoparticles," Materials Chemistry and Physics, vol. 105, no. 2-3, pp. 419-425, 2007.

[8] T. Liu, D. Li, D. Yang, and M. Jiang, "An improved seedmediated growth method to coat complete silver shells onto silica spheres for surface-enhanced Raman scattering," Colloids and Surfaces A, vol. 387, no. 1-3, pp. 17-22, 2011.

[9] S. L. Westcott and N. J. Halas, "Electron relaxation dynamics in semicontinuous metal films on nanoparticle surfaces," Chemical Physics Letters, vol. 356, no. 3-4, pp. 207-213, 2002.

[10] D. A. Slanac, L. Li, A. Mayoral et al., "Atomic resolution structual insights into PdPt nanoparticle-carbon interactions for the design of highly active and stable electrocatalysts," Electrochimica Acta, vol. 64, pp. 35-45, 2012.

[11] Q. Zhou, H. Chen, and Y. Wang, "Region-selective electroless gold plating on polycarbonate sheets by UV-patterning in combination with silver activating," Electrochimica Acta, vol. 55, no. 7, pp. 2542-2549, 2010.
[12] J. Hu, W. Li, J. Chen, X. Zhang, and X. Zhao, "Novel plating solution for electroless deposition of gold film onto glass surface," Surface and Coatings Technology, vol. 202, no. 13, pp. 2922-2926, 2008.

[13] K. Miyoshi, S. Fujikawa, and T. Kunitake, "Fabrication of nanoline arrays of noble metals by electroless plating and selective etching process," Colloids and Surfaces A, vol. 321, no. 1-3, pp. 238-243, 2008.

[14] Y. Kobayashi, Y. Tadaki, D. Nagao, and M. Konno, "Deposition of gold nanoparticles on silica spheres by electroless metal plating technique," Journal of Colloid and Interface Science, vol. 283, no. 2, pp. 601-604, 2005.

[15] V. P. Menon and C. R. Martin, "Fabrication and evaluation of nanoelectrode ensembles," Analytical Chemistry, vol. 67, no. 13, pp. 1920-1928, 1995.

[16] L. M. Liz-Marzán, M. Giersig, and P. Mulvaney, "Synthesis of nanosized gold-silica core-shell particles," Langmuir, vol. 12, no. 18, pp. 4329-4335, 1996.

[17] C. F. Bohren and D. F. Huffman, Absorption and Scattering of Light by Small Particles, John Wiley \& Sons, New York, NY, USA, 1983. 

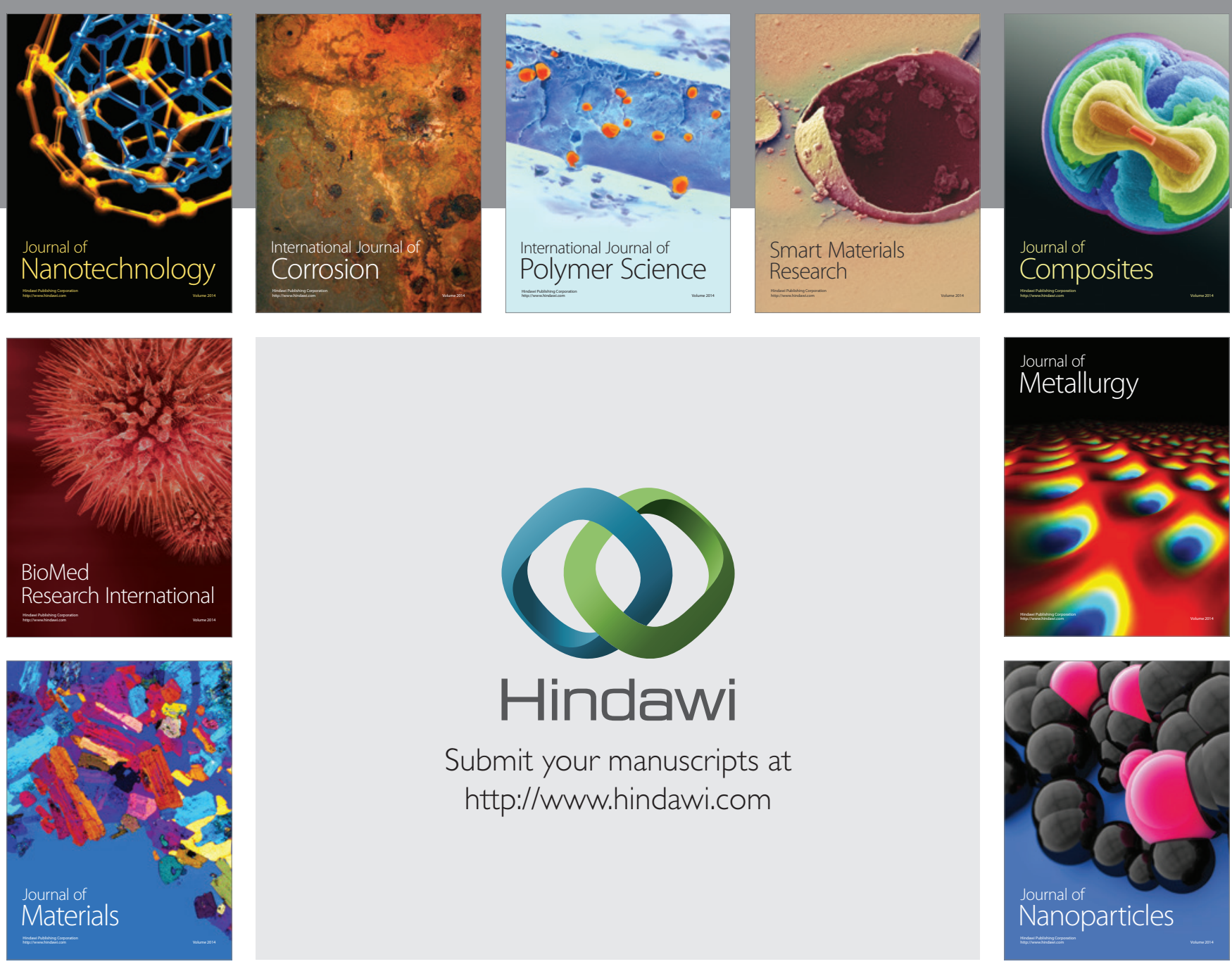

Submit your manuscripts at http://www.hindawi.com
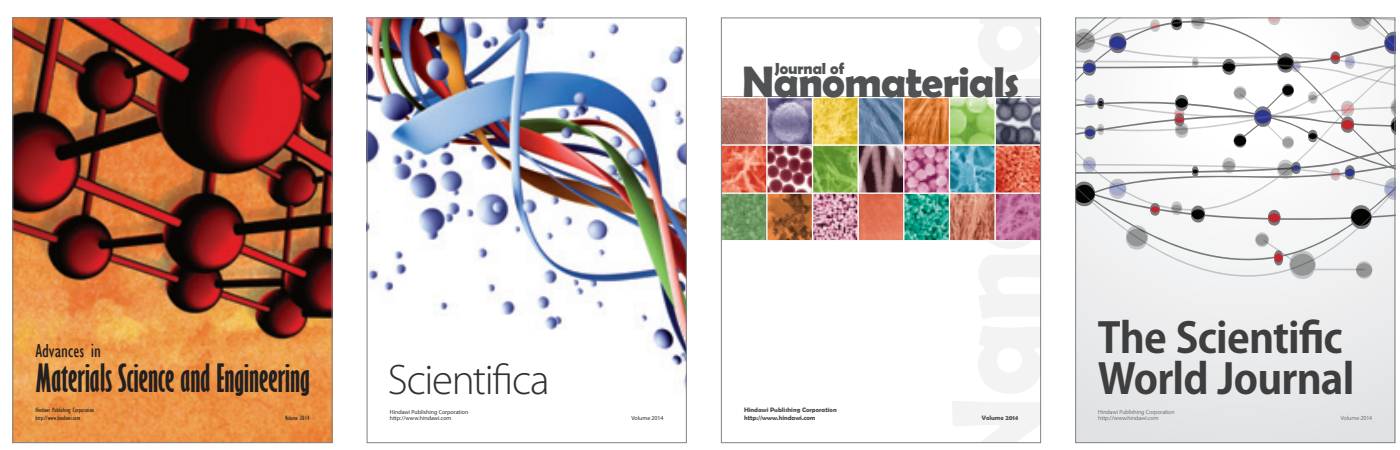

\section{The Scientific World Journal}
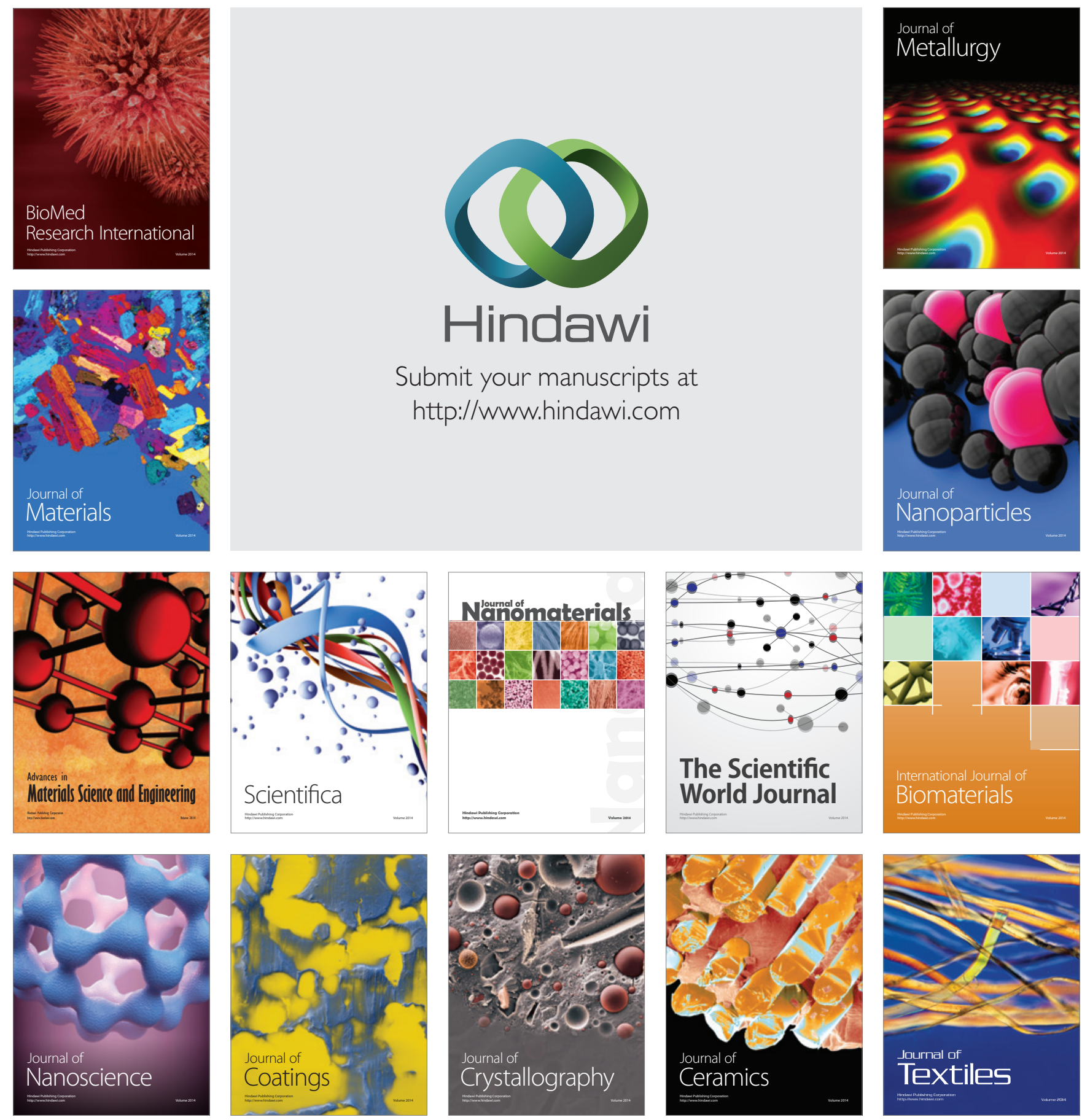